\title{
Communications
}

\section{Free-Field Propagation of Localized Pulses}

\section{EGON MARX}

\begin{abstract}
Incident transient fields obey homogeneous Maxwell equations and are assumed to be a known input to the integral equations of electromagnetic scattering. These fields can be specified by two solenoidal fields for $\vec{E}$ and $\vec{B}$ at the initial time, and they can be computed at other times using the Green function for the scalar wave equation. Equations are derived that are then used in a computer program to do the integrations over the information-collecting sphere. Sample calculations show that a modulated pulse keeps its shape much better than a simple pulse.
\end{abstract}

\section{INTRODUCTION}

To compute the fields of an electromagnetic wave scattered by an object, it is customary to decompose the fields outside the scatterer into incident and scattered parts. If the fields are monochromatic, the incident fields are arbitrary solenoidal fields. If the incident fields form a plane-wave pulse, they propagate without changing shape and are trivially determined by their initial values. If the incident pulse is localized and has finite energy, the fields obey the homogeneous Maxwell equations, but the solution is generally not trivial. These fields can be specified by initial or boundary conditions, but their values at other times or points are also required to solve for the scattered fields. Here we address the initial-value problem for free fields. The propagation of free fields is of interest also in the design and representation of devices, when monochromatic or plane waves are not adequate approximations.

One possible approach to the representation of transient localized electromagnetic fields is the use of approximate solutions of Maxwell's equations, such as those related to the paraxial approximation [1]-[3]. In this note, we discuss the numerical computation of exact solutions after they are reduced to integrals of the initial values of the fields over the information-collecting sphere. We derive the integrals for the fields, we briefly describe a specific computer program we developed, and we discuss the effects of modulation through an example.

\section{Propagation of Localized Pulses}

We assume that fields $\vec{E}_{0}$ and $\vec{B}_{0}$ are given at the initial time, $t=0$, throughout space, subject to the constraints

$$
\nabla \cdot \vec{E}_{0}(\vec{x})=0, \quad \nabla \cdot \vec{B}_{0}(\vec{x})=0 .
$$

The general solution of Maxwell's equations [4] in terms of the retarded Green function for the scalar wave equation, $G_{R}^{(0)}$, in the absence of sources and boundaries, reduces to

$$
\begin{aligned}
\vec{E}(\vec{x}, t)=-\int_{V^{\prime}} d V^{\prime} & {\left[\frac{1}{c^{2}} \vec{E}_{0}\left(\vec{x}^{\prime}\right) \frac{\partial G_{R}^{(0)}\left(\vec{x}, t ; \vec{x}^{\prime}, 0\right)}{\partial t^{\prime}}\right.} \\
& \left.-\vec{B}_{0}\left(\vec{x}^{\prime}\right) \times \nabla^{\prime} G_{R}^{(0)}\left(\vec{x}, t ; \vec{x}^{\prime}, 0\right)\right],
\end{aligned}
$$

Manuscript received March 8, 1985; revised May 20, 1985.

The author is with the National Bureau of Standards, Gaithersburg, MD 20899 .

$$
\begin{aligned}
\vec{B}(\vec{x}, t)=-\frac{1}{c^{2}} \int_{V} d V^{\prime} & {\left[\vec{E}_{0}\left(\vec{x}^{\prime}\right) \times \nabla^{\prime} G_{R}^{(0)}\left(\vec{x}, t ; \vec{x}^{\prime}, 0\right)\right.} \\
& \left.+\vec{B}_{0}\left(\vec{x}^{\prime}\right) \frac{\partial G_{R}^{(0)}\left(\vec{x}, t ; \vec{x}^{\prime}, 0\right)}{\partial t^{\prime}}\right],
\end{aligned}
$$

where $V$ is all of space. The retarded Green function is

$$
G_{R}^{(0)}\left(\vec{x}, t ; \vec{x}^{\prime}, t^{\prime}\right)=\frac{\delta\left(t-t^{\prime}-R / c\right)}{4 \pi R},
$$

where $\vec{R}=\vec{x}-\vec{x}^{\prime}$ and $R=|\vec{R}|$. Substitution into (2) and (3) reduces the integrals to surface integrals [5],

$$
\begin{aligned}
& \vec{E}(\vec{x}, t)=\frac{1}{4 \pi} \int d \Omega\left[\vec{E}_{0}-\vec{R} \cdot \nabla^{\prime} \vec{E}_{0}\right. \\
& \left.+\left(2 c \vec{B}_{0}-\vec{R} \cdot \nabla^{\prime} c \vec{B}_{0}\right) \times \vec{R}\right]_{R=c t}, \\
& \left.\left.-\vec{R} \cdot \nabla^{\prime} \vec{E}_{0}\right) \times \vec{R}\right]_{R=c l} .
\end{aligned}
$$

The integrations are carried out over the information-collecting sphere of radius $R=c t$ centered at the observation point $\vec{x}$; the arguments $\vec{x}^{\prime}$ of the fields are expressed in terms of the spherical coordinates $\theta$ and $\phi$, and the surface element is reduced to the element of solid angle $\mathrm{d} \Omega$. If $\vec{E}_{0}$ and $\vec{B}_{0}$ are given analytically, the gradients can be found by differentiation; otherwise, they have to be determined numerically.

\section{Program "Ball"}

We have written a program, described in [5], to perform the operations indicated in (5) and (6). We specify the initial fields by setting

$$
\begin{gathered}
\vec{E}_{0}(\vec{x})=A \exp \left[-\kappa\left(x^{2}+y^{2}\right)\right](y \hat{i}-x \hat{j}) \Phi^{\prime}(z), \\
c \vec{B}_{0}(\vec{x})=A \exp \left[-\kappa\left(x^{2}+y^{2}\right)\right]\left\{(x \hat{i}+y \hat{j}) \Phi^{\prime}(z)\right. \\
\left.-2 \hat{k}\left[1-\kappa\left(x^{2}+y^{2}\right)\right] \Phi(z)\right\},
\end{gathered}
$$

which are not too different from the fields of a plane-wave pulse propagating in the $z$-direction, have essentially a Gaussian profile in the $x y$-plane, and satisfy the constraints (1) for any $\Phi$. The field $\vec{E}_{0}$ vanishes on the $z$-axis, but this is not a necessary condition.

The integration over the surface of the information-collecting sphere can be restricted to those regions where the initial data are not negligible. These regions are inside a finite cylinder of radius proporational to $1 / \sqrt{\kappa}$ and with top and bottom planes determined by $\Phi$.

The output of the main program includes the components of the fields, which are stored in a file for further processing. For instance, the energy density can be represented in a three-dimensional plot as a function of $x$ and $z$, or it can be scaled to 256 gray levels and displayed as an intensity image on the screen of an image-processing system. We have generated a sequence of such images and photographed them to produce a movie that shows the time development of the pulse. 

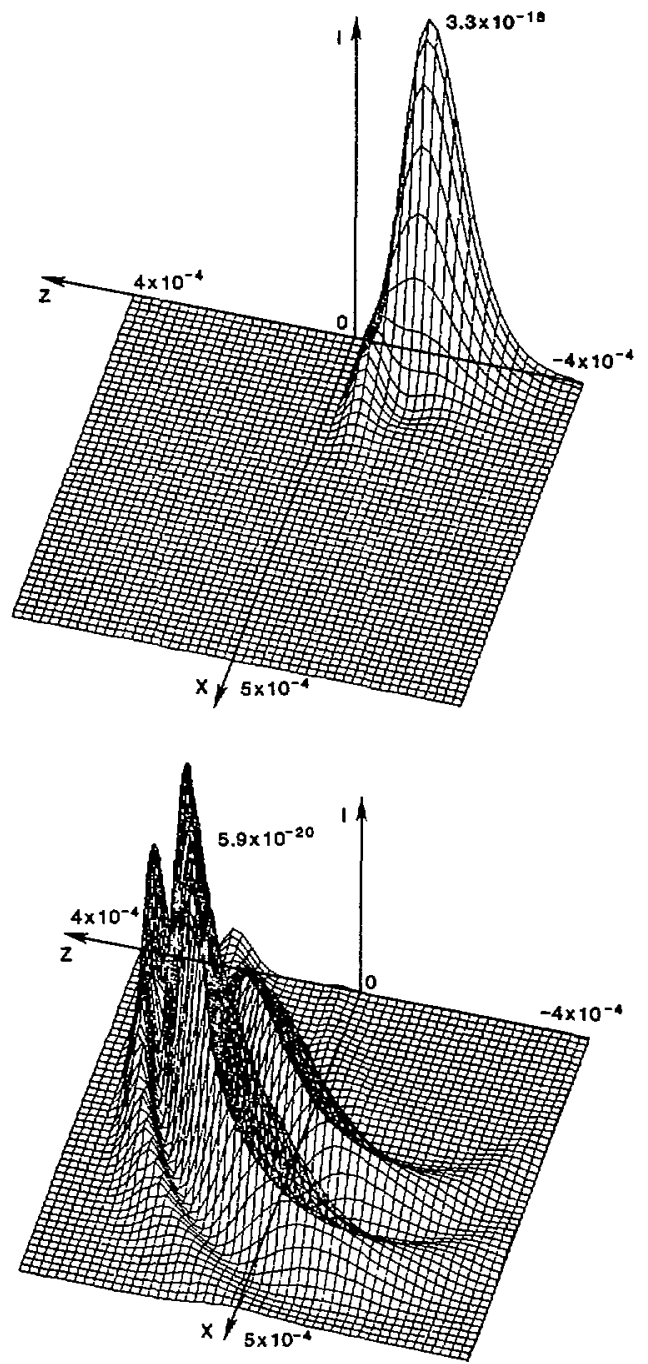

Fig. 1. Energy density of a simple pulse as a function of $x$ and $z$ at times $t=$ 0 and $t=1.2 \times 10^{-12} \mathrm{~s}$. There is cyclindrical symmetry of the fields about the $z$-axis, and the part of the energy plot for negative $x$ is a mirror image of the part shown. Distances are shown in meters and intensities are shown in arbitrary units; the intensity scale differs from one plot to the other to show detail in the propagated pulse, which has a peak that is smaller than that of original pulse by a factor of 56 .

Conservation of energy and momentun is used in an ancillary program to verify the accuracy of the computed fields.

\section{MODULATION}

The program was applied specifically to a double exponential,

$$
\Phi_{1}(z)=z^{2}[\exp (\alpha z)-\exp (\beta z)] \Theta(-z),
$$

where $\theta$ is the unit step function, $\alpha=2 \times 10^{4} \mathrm{~m}^{-1}, \beta=3 \times 10^{4}$ $\mathrm{m}^{-1}$, and the factor $z^{2}$ was added to improve the smoothness at $z=$ 0 . A plot of the energy density (proportional to $\vec{E}^{2}+c^{2} \vec{B}^{2}$ ) as a function of position is shown in Fig. 1 at $t=0$ and $t=1.2 \times 10^{-12}$ $s$. The pulse travels essentially in the $z$-direction but there is considerable radial spread. The space and time variables can be scaled simultaneously to represent other pulses of the same shape.

Another example of $\Phi$ was

$$
\Phi_{2}(z)=[\exp (\alpha z)-\exp (\beta z)](\sin k z) \Theta(-z) .
$$

The corresponding plots are shown in Fig. 2. The radial spread is much decreased by superimposing a few oscillations of the underly-
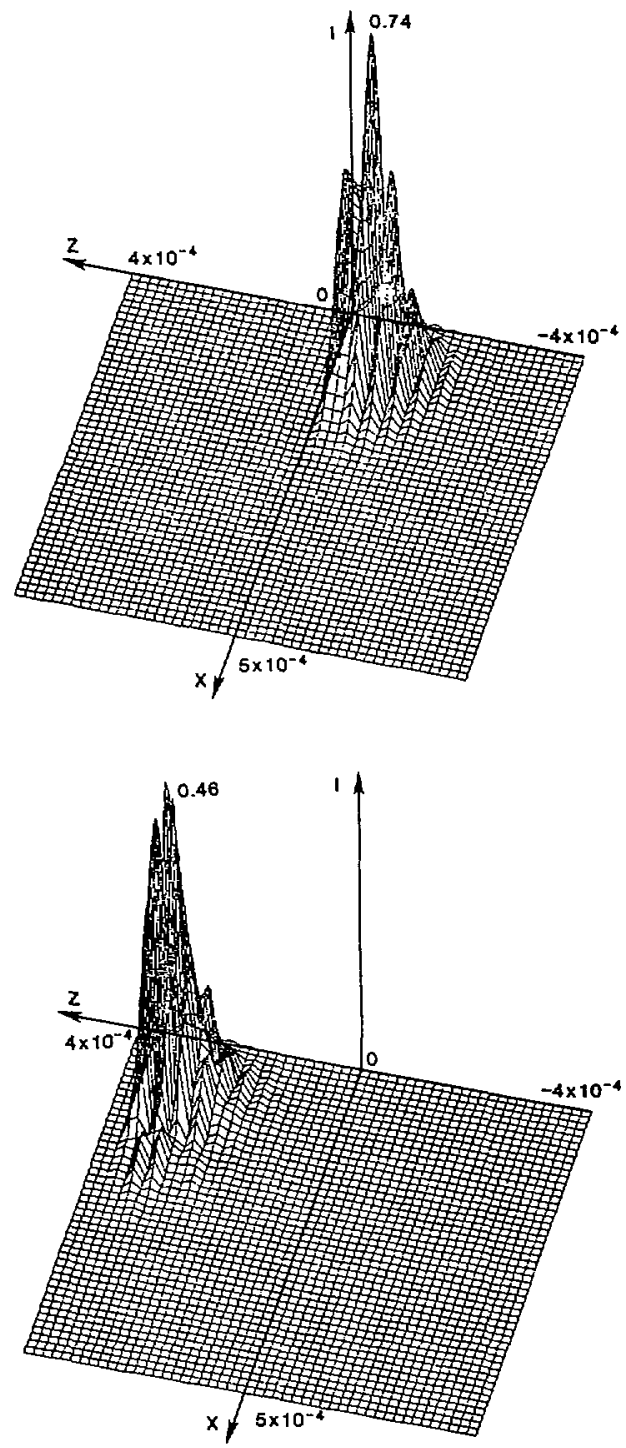

Fig. 2. Energy density of a modulated pulse. The times and spatial regions are the same as in Fig. 1, while the intensity units are different. The intensity scale also differs from one plot to the other.

ing carrier $\left(k=1 \times 10^{5} \mathrm{~m}^{-1}\right)$. In Figs. 1 and 2 , the intensity of the fields is arbitrary, and the vertical scales differ from plot to plot to show the form of the propagated pulse. The numbers on the plots give the value of the peak energy densities. For the simple pulse in Fig. 1, the peak of the propagated pulse is about 0.02 of the initial peak value. The peak of the modulated pulse in Fig. 2 is about 0.6 of the initial peak value after the same time interval.

This inverse relationship between the spreading of the pulse and the underlying frequency can also be seen in the analytical treatment in [2].

\section{CONCLUSION}

The fields at a point $\vec{x}$ and at time $t$ can be computed in a straightforward manner to allow us, for instance, to determine the incident fields in a scattering problem. A simple pulse spreads in the directions perpendicular to the direction of propagation, but even a small amount of oscillation to form a modulated pulse results in a much better defined shape and direction of propagation.

These calculations are rigorous and the accuracy of the results depends only on the numerical procedures used to do the integrations in (5) and (6). 


\section{REFERENCES}

[1] J. Cooper, "Exact and approximate pulsed beam solutions of Maxwell's equations," Math. Meth. Appl. Sci., in press.

[2] J. Cooper and E. Marx, "Free-space propagation of ultra-short light pulses," J. Opt. Soc. Am., in press.

[3] H. Kogelnik and T. Li, "Laser beams and resonators," Proc. IEEE, vol. 54, pp. 1312-1329, 1966.

[4] E. Marx, "Integral equations for transient electromagnetic fields," Nat. Bur. Stand. U.S. Tech. Note 1157, Feb. 1982.

[5] - - "Free-space propagation of light pulses," Nat. Bur. Stand. U.S. NBSIR 84-2835, May 1984.

\section{A Note on Pulse Distortion by Ionospheric Reflection}

\section{LEWIS E. VOGLER}

\begin{abstract}
An expression, suitable for a small desk-top computer, is presented to calculate the time domain envelope of a rectangular pulse reflected from a sech ${ }^{2}$ model ionosphere and observed at the output of a simple receiver bandpass filter. lonospheric, source pulse, and filter parameters can be varied to estimate distortion characteristics of the received pulse.
\end{abstract}

\section{INTRODUCTION}

Investigations concerning the distortion of ionospherically reflected pulses continue to be of interest in such fields as digital communication and navigation systems and pulse signature detection [1]. Elaborate computer programs can be written to evaluate numerically various system and propagation configurations, but it is also helpful to have a simpler model that can estimate characteristics of the received pulse. This note suggests an expression derived from a $\operatorname{sech}^{2}$ model ionosphere, a rectangular source pulse, and a simple $R L C$ series circuit receiver filter. The equation can be evaluated with a small desk-top computer and is applicable to a variety of ionospheric layer conditions.

The time domain waveform $E_{r}(t)$ of a pulse that is reflected from the ionosphere and observed at the output terminals of a receiver bandpass filter can be expressed as

$$
E_{r}(t)=\left(E_{0} / 2 \pi\right) \int_{-\infty}^{+\infty} S(\omega) R(\omega) B(\omega) e^{i \omega t} d \omega
$$

where $E_{0}$ is an amplitude constant determined from the source, $\omega=$ $2 \pi f$ denotes the electromagnetic (angular) frequency, $S(\omega)$ and $B(\omega)$ are the frequency spectra of the source pulse and bandpass filter, respectively, and $R(\omega)$ represents the ionospheric reflection coefficient.

A recent paper [2] has investigated the impulse response for an ionospheric model characterized by a $\operatorname{sech}^{2}$ electron density height profile and constant values of electron collision frequency and vertical component (only) of the earth's magnetic field. With $\omega_{p}$ denoting the penetration frequency, and $z_{m}$ and $\sigma$ the maximum density height and width parameter of the ionospheric layer, the

Manuscript received January 2, 1985; revised May 13, 1985.

The author is with the Insitute for Telecommunication Sciences, National Telecommunications and Information Administration, U.S. Department of Commerce, Boulder, CO 80303. refractive index $\boldsymbol{n}$ for a $\operatorname{sech}^{2}$ model is given by

$$
\begin{array}{ll}
n_{\epsilon}^{2}=1-\left\{\left(\omega_{p} / \omega\right)^{2} / b_{\epsilon}\right\} \operatorname{sech}^{2}\left\{\left(z-z_{m}\right) / 2 \sigma\right\}, & \\
& (\epsilon= \pm 1) \\
b_{\epsilon}=1+\left(\epsilon \omega_{H}-i \nu\right) / \omega, &
\end{array}
$$

where $\nu$ and $\omega_{H}$ are the collision frequency and gyro frequency, respectively. For oblique incidence we also define $C \equiv \cos \theta$ with $\theta$ being the angle of incidence that the wave-normal in the $x-z$ plane makes with the vertical $z$ axis in a Cartesian coordinate system.

Solutions for the field components were derived (exact for vertical incidence-approximate for oblique incidence), and two reflection coefficients were defined in terms of the linearly polarized incident and reflected $E$-fields:

1) $R_{v}$ : the ratio of the reflected $x$ to the incident $x$ component;

2) $R_{h}$ : the ratio of the reflected $y$ to the incident $x$ component.

The reflection coefficients involve gamma functions of complex argument, and numerical methods can be used to evaluate (1) and show the detailed behavior of the received pulse. However, the envelope of the pulse often provides a more practical means of presenting significant pulse shape characteristics.

In many cases an expressions for the absolute value of the envelope of (1) can be obtained by applying the method of stationary phase. This results in

$$
E_{r} / E_{0} \sim \sum_{\omega_{r}}\left|f\left(\omega_{t}\right) /\left\{2 \pi \phi^{\prime \prime}\left(\omega_{t}\right)\right\}^{1 / 2}\right| e^{i\left\{\omega_{t} t+\phi\left(\omega_{t}\right)\right\}},
$$

where

$$
\begin{gathered}
f(\omega)=S(\omega) R(\omega) B(\omega), \\
\phi(\omega)=\arg S(\omega)+\arg R(\omega)+\arg B(\omega),
\end{gathered}
$$

and $\omega_{t}$ represents the value (or values) for which

$$
(d / d \omega)[\omega t+\phi(\omega)]=t+\phi^{\prime}(\omega)=0 .
$$

In general the summation in (3) should be over all values of $\omega_{t}$. However, if the only significant roots of (5) are the pair, $\pm \omega_{t}$, and if the Hermitian relationship holds, i.e., $f(-\omega)=f^{*}(\omega)$, then (3) becomes

$E_{r} / E_{0} \sim(2 / \pi)^{1 / 2}\left|S\left(\omega_{t}\right) R\left(\omega_{t}\right) B\left(\omega_{t}\right) /\left\{\phi^{\prime \prime}\left(\omega_{t}\right)\right\}^{1 / 2}\right| \cos \left\{\omega_{t} t+\phi\left(\omega_{t}\right)\right\}$,

where

$\phi^{\prime \prime}\left(\omega_{t}\right)=\left.\left(d^{2} / d \omega^{2}\right)[\arg S(\omega)+\arg R(\omega)+\arg B(\omega)]\right|_{\omega=\omega_{t}} \neq 0$.

The factor multiplying the cosine term in (6) now provides an approximation to the positive portion of the envelope of (1).

In presenting results for the $\operatorname{sech}^{2}$ model, it is convenient to introduce the quantities

$$
\begin{gathered}
\zeta=\alpha \omega, T=\left\{t-2(C / c)\left(z_{m}-z_{1}\right)\right\} / \alpha, \\
\alpha=2(\sigma / c) C, \delta=2(\sigma / c) \omega_{p},
\end{gathered}
$$

where $c$ is the speed of light and $z_{1}$ is the height to which the reflection coefficient is referred. Then, as shown in [2], the envelope of the impulse response obtained by setting $S(\omega)$ and $B(\omega)$ in (1) to 